\title{
Probe-based confocal laser endomicroscopy (PCLE) is a suitable method for extrapulmonary high grade neuroendocrine rectal carcinoma (HGNEC) evaluation
}

This article was published in the following Dove Press journal: OncoTargets and Therapy

\author{
Renato Cannizzaro' \\ Stefania Maiero' \\ Mara Fornasarig' \\ Vincenzo Canzonieri ${ }^{2}$ \\ Raffaella Magris' \\ Giovanni Guarnieri' \\ Martina Urbani ${ }^{3}$ \\ Angela Buonadonna ${ }^{4}$ \\ Tanja Baresic ${ }^{5}$ \\ Paola Spessotto ${ }^{6}$ \\ 'Oncological Gastroenterology, Centro di \\ Riferimento Oncologico di Aviano (CRO) \\ IRCCS, Aviano, Italy; ${ }^{2}$ Pathology, Centro di \\ Riferimento Oncologico di Aviano (CRO) \\ IRCCS, Aviano, Italy; ${ }^{3}$ Oncologic Radiation \\ Therapy and Diagnostic Imaging, Centro di \\ Riferimento Oncologico di Aviano (CRO) \\ IRCCS, Aviano, Italy; ${ }^{4}$ Medical Oncology, \\ Centro di Riferimento Oncologico di \\ Aviano (CRO) IRCCS, Aviano, Italy; \\ ${ }^{5}$ Nuclear Medicine, Centro di Riferimento \\ Oncologico di Aviano (CRO) IRCCS, \\ Aviano, Italy; 'Molecular Oncology, \\ Department of Translational Research, \\ Centro di Riferimento Oncologico di \\ Aviano (CRO) IRCCS, Aviano, Italy
}

Corresponcence: Renato Cannizzaro Oncological Gastroenterology, Centro di Riferimento Oncologico di Aviano (CRO) IRCCS, Via Franco Gallini 2, 3308I

Aviano, Italy

Tel +39043465 928I

Fax +390434659515

Email rcannizzaro@cro.it

\begin{abstract}
The potential role of the probe-based confocal laser endomicroscopy (pCLE) has been analyzed in different pathologic conditions of the gastrointestinal tract. Here, we analyzed a case of extrapulmonary high grade neuroendocrine rectal carcinoma (HGNEC) using, for the first time, the pCLE system. A 72-year old man was diagnosed with an $8 \mathrm{~cm}$ diameter rectal HGNEC by standard colonoscopy integrated with the pCLE system. The diagnosis of neuroendocrine carcinoma was confirmed by immunohistochemical analyses. By using the pCLE system, we well defined and resolved vascular structures and mucosal architecture. An altered mucosal pattern and vascular defects, peculiar for HGNEC, were observed at high magnification, allowing the identification of a pattern which was quite different from that observed in poorly differentiated adenocarcinomas (PDA) where tissues appear darker, very irregular, even if glandular structures can still be recognized. This underlines the usefulness of pCLE in discriminating HGNECs from PDAs. In conclusion, pCLE could represent a valid and helpful method for in vivo HGNEC diagnosis, allowing prompt and careful management of the patient.
\end{abstract}

Keywords: probe-based confocal endomicroscopy, neoangiogenesis, extrapulmonary high grade neuroendocrine carcinoma, poorly differentiated adenocarcinoma

\section{Introduction}

Extrapulmonary high grade neuroendocrine carcinomas (HGNECs) are rare but very aggressive tumors that can be found throughout the body, however, they are usually located in the gastrointestinal (GI) and genitourinary tracts. ${ }^{1}$ HGNEC is different from poorly differentiated carcinoma with neuroendocrine features, which is not a true HGNEC but an aggressive variant of adenocarcinoma or squamous cell carcinoma that should be managed as such. Since most HGNECs are diagnosed at advanced stage, staging is performed using the standard techniques of endoscopic ultrasound, computed tomography (CT) and positron emission tomography (PET) scan of the abdomen, thorax, and pelvis. ${ }^{1,2}$ The most common sites of metastatic spread are the liver and regional lymph nodes, followed by the lung and bone. ${ }^{1,3}$ Rectal neuroendocrine tumors (NETs) represent $2 \%$ of all rectal tumors, and among these only $5 \%$ are bigger than $20 \mathrm{~mm} .{ }^{4}$ Moreover, poorly differentiated rectal NETs represent $12 \%$ of all poorly differentiated gastro-entero-pancreatic NETs, therefore rectal HGNECs are very rare.,5 They are very aggressive and almost always 
metastatic at presentation. ${ }^{6}$ These features require that an accurate diagnosis should be as rapid as possible for the best management of the patient.

The probe-based confocal laser endomicroscopy (pCLE) system is a highly enhanced endoscopic technique that provides high quality imaging of the tissue, with a resolution of approximately 1 micron of the mucosal layer. $^{7,8}$ The main clinical application for which pCLE was developed includes the real-time histopathological diagnosis of GI lesions. ${ }^{9,10}$ However, in recent years, additional potential application has been demonstrated to record vascular changes and alterations, offering the possibility to obtain information on the morphological and functional characteristics of GI tumor vessels in real time. $^{11,12}$

Here we describe for the first time, the use of pCLE as a powerful diagnostic technique in an HGNEC patient.

\section{Case report}

Endoscopy procedures and PCLE analyses pCLE analyses were carried out with GastroFlex UHD probe (Cellvizio, Mauna Kea Technology, Paris, France) during colonoscopy (Olympus series 180, Olympus Corporation, Tokyo, Japan) and immediately before endoscopic ultrasonography (Olympus series 160), as previously described. ${ }^{12}$ Images and sequences of the normal and neoplastic mucosa were taken and the conventional bioptic samples obtained with macrobiopsy (COOK Medical, Ireland) at the end of examination. Images were recorded within the first 10 minutes following intravenous injection of fluorescein ( $5 \mathrm{~mL}$ of a $10 \%$ solution). By using intravenously administered fluorescein sodium, ${ }^{13}$ images of colonic mucosa as well as vascular network can be clearly detected and high video quality with a direct visualization on a single erythrocyte scale easily provided. pCLE recordings were performed for at least 3 minutes. The mucosal architecture, vessel morphology, and the efficiency of the blood flow were evaluated. The images were digitally stored and independently reviewed with the dedicated software package (Cellvizio Viewer, Mauna Kea Technologies) by the endoscopist who performed pCLE, by another expert endoscopist, and by a single investigator who was blinded to any clinical, endoscopic, or histopathological information. The evaluation was considered valid if all three investigators were concordant.

By using the pCLE system it is possible to discriminate the typical aberrant features characterizing the tumor- associated blood vessels. The vascular architecture of all the vessels is generally abnormal, being mostly represented by enlarged, distorted, and highly permeable microvessels with altered erythrocyte flow. Based on these observations, we have generated an "angiogenic score" ranging from 0 (normal vasculature) to 4 (extremely aberrant and non-functional vasculature). The angiogenic score was assigned on the basis of the presence of tortuous and large-sized vessels, the vessels' leakage, and the presence of defective flow as previously described. ${ }^{12}$

\section{Presenting concerns}

A 72-year old man presented with abdominal pain, alternated diarrhea and constipation, fatigue, weight loss, and discomfort in the last 2 months and positive hemoccult. He had no other systemic diseases, no family history of cancer, and physical examination was negative.

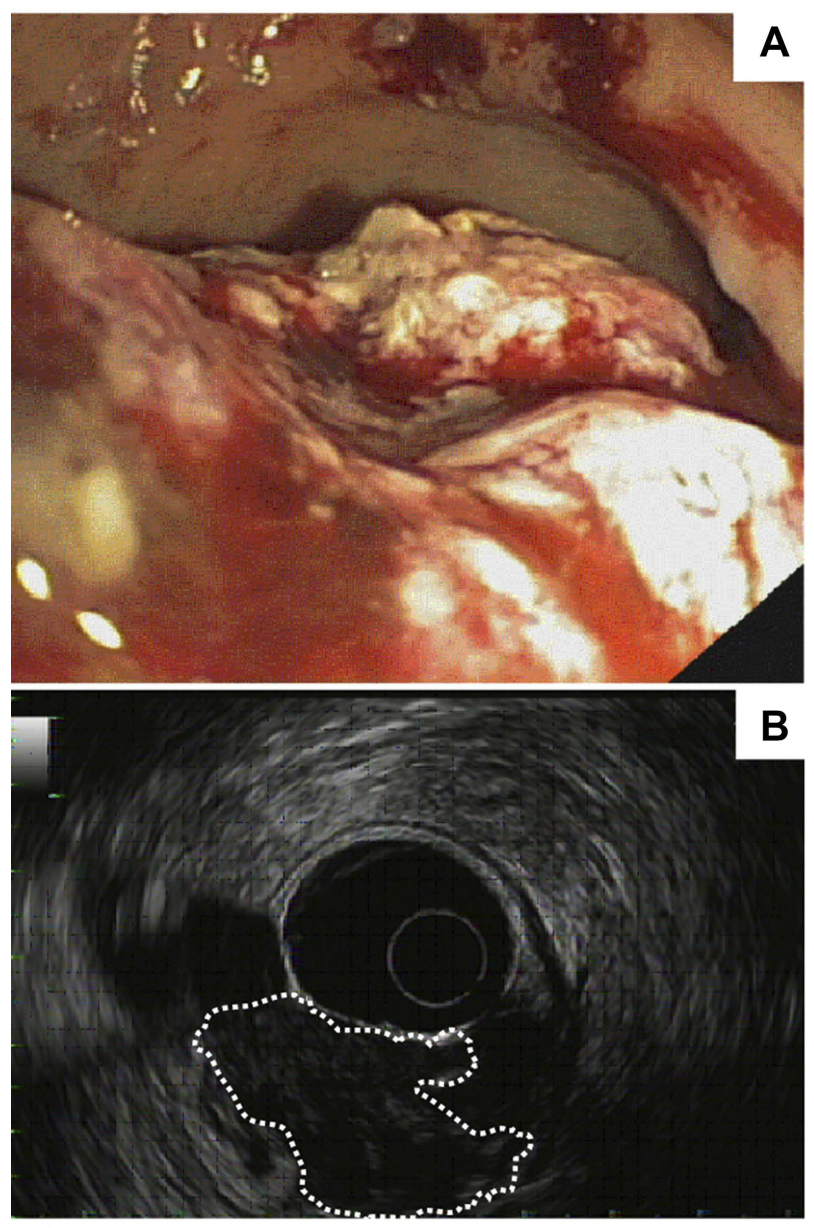

Figure I Rectal HGNEC endoscopic (A) and EUS (B) views. (B) Tumor mass is highlighted by white dotted line.

Abbreviations: HGNEC, high grade neuroendocrine rectal carcinoma; EUS, endoscopic ultrasound. 

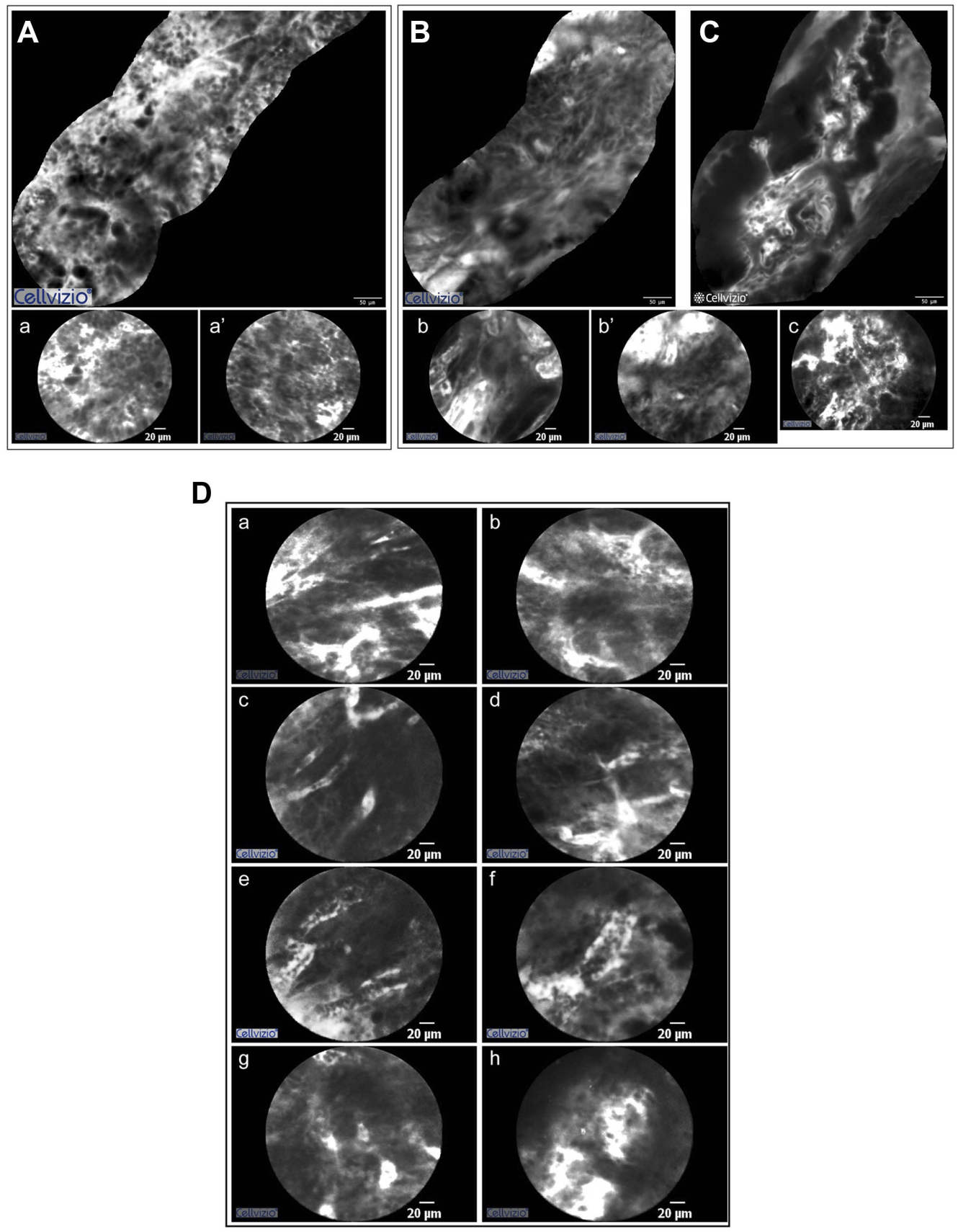

Figure 2 Mosaic reconstructions (A, B, and C) and images (a, a', b, b', and c) obtained with pCLE in HGNEC (A, a and a') and in two poorly differentiated adenocarcinoma patients (B, b, b' and $\mathbf{C}, \mathrm{c}$ ). The neoplastic mucosa in the HGNEC patient is irregular. The well defined and resolved cellular architecture allows the recognition of a small cell type pattern. The other two patients displayed highly irregular mucosa but small cell type pattern was not observed. Areas with very dark epithelium were more evident in poorly differentiated adenocarcinoma samples than in HGNEC. Glandular structure was detectable in poorly differentiated adenocarcinoma (b). (D) Vascular pattern in HGNEC patient. Dilated, large vessels were easily detectable in highly vascularized areas (a-f). These vessels are often characterized by defective blood flow. Leakage ( $g$ ) and tortuous vascular structures (h) were present.

Abbreviations: HGNEC, high grade neuroendocrine rectal carcinoma; pCLE, probe-based confocal laser endomicroscopy.

\section{Clinical findings}

A colonoscopic evaluation showed an $8 \mathrm{~cm}$ diameter noncircumferential raised lesion at rectum with superficial necrosis (Figure 1A). During the same session the patient underwent endoscopic ultrasound which showed a rectal neoplasm which was hypoechoic, inhomogeneous with anechoic areas infiltrating all parietal layers and with the absence of peri-rectal lymphadenopathy (Figure 1B). pCLE recording was also performed during the same session resulting in a real-time imaging of more than 2,000 


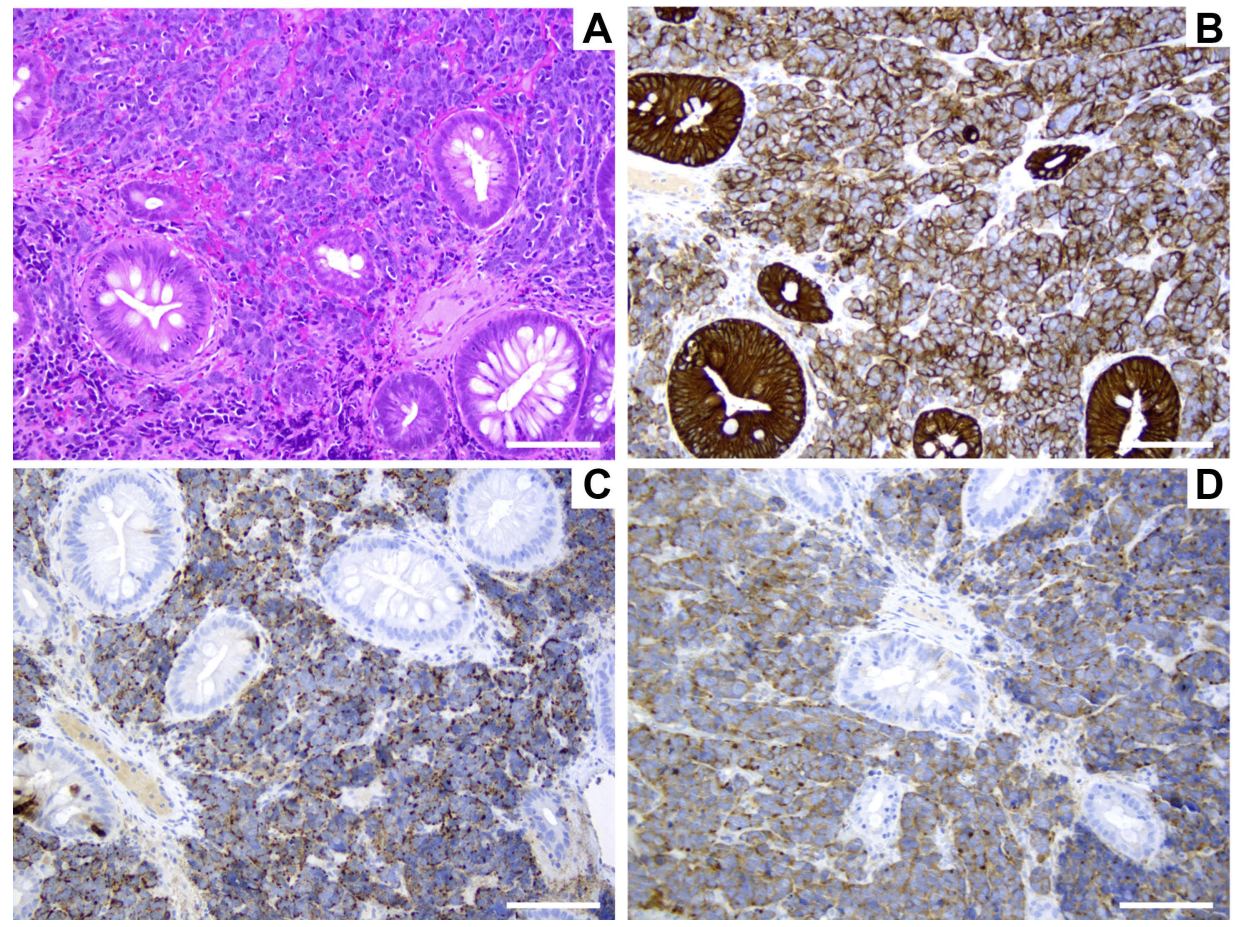

Figure 3 Histopathological examination of the HGNEC patient. Representative images of H\&E staining (A) and of immunostaining of keratin (B), chromogranin A (C), and synaptophysin (D). Scale bar $=100 \mu \mathrm{m}$.

Abbreviation: HGNEC, high grade neuroendocrine rectal carcinoma.

frames off-line analyzed. We also obtained, by video mosaicing function provided by the software (Cellvizio Viewer), the reconstruction of tissue panoramas. The principal feature of the altered mucosal architecture detectable by pCLE was the presence of a huge amount of small cells (Figure 2A, a, a'), a typical and very peculiar cellular pattern for HGNEC. ${ }^{1,2}$ The vasculature pattern well defined and resolved by pCLE allowed us to describe for the first time, the characteristic alterations found in an HGNEC blood network (Figure 2), showing a prominent high vessel density referring to a 4 score according to angiogenesis score described by Spessotto et al. ${ }^{11,12}$ The most frequent and represented vascular anomaly was the presence of large vessels with defects in blood flow. In the supplementary Videos S1, above all, the presence of enlarged vessels can be appreciated; in addition, the sequences of Videos S2 clearly document slowing down of blood flow. Another example of defective flow is reported in Videos S3. We also observed the abundance of very irregular, tortuous, and dilated vessels, with no orientation to adjunct tissue and some regions of leakage indicating high vessel permeability (Figure 2D and Videos S1 and S2). This pattern was quite different from poorly differentiated adenocarcinomas (PDA) where tissues appear darker, very irregular, even if glandular structures can still be recognized (Figure 2B, b, b' and Figure 2C, c). PDA patients generally display an angiogenesis score of 3 . We evaluated five PDA patients who did not display aberrations in blood flow (data not shown).

The positive staining for both chromogranin and synaptophisin markers revealed by immunohistochemistry confirmed the diagnosis of neuroendocrine carcinoma (Figure 3). Abdominal CT scan showed an increased liver size with multiple disseminated hypodense lesions, and lymph nodes at celiac and lombo-aortic region. Moreover, lower abdominal CT showed a solid and concentric thickening at sigma-rectum junction in pre-sacral region (Figure 4A). CT/PET scan confirmed multiple liver metastases, the presence of lymph nodes at celiac, lomboaortic, and hepatic hilum region and showed significant 18-F fluorodeoxyglucose uptake at vertebral column, shoulder blades, chest and hip bone (Figure 4B). Platinum-based chemotherapy, the best option for disease control in this metastatic setting, was promptly started.

\section{Discussion}

HGNECs constitute a series of aggressive malignancies. Colorectal HGNECs are a rare histological subtype, with 


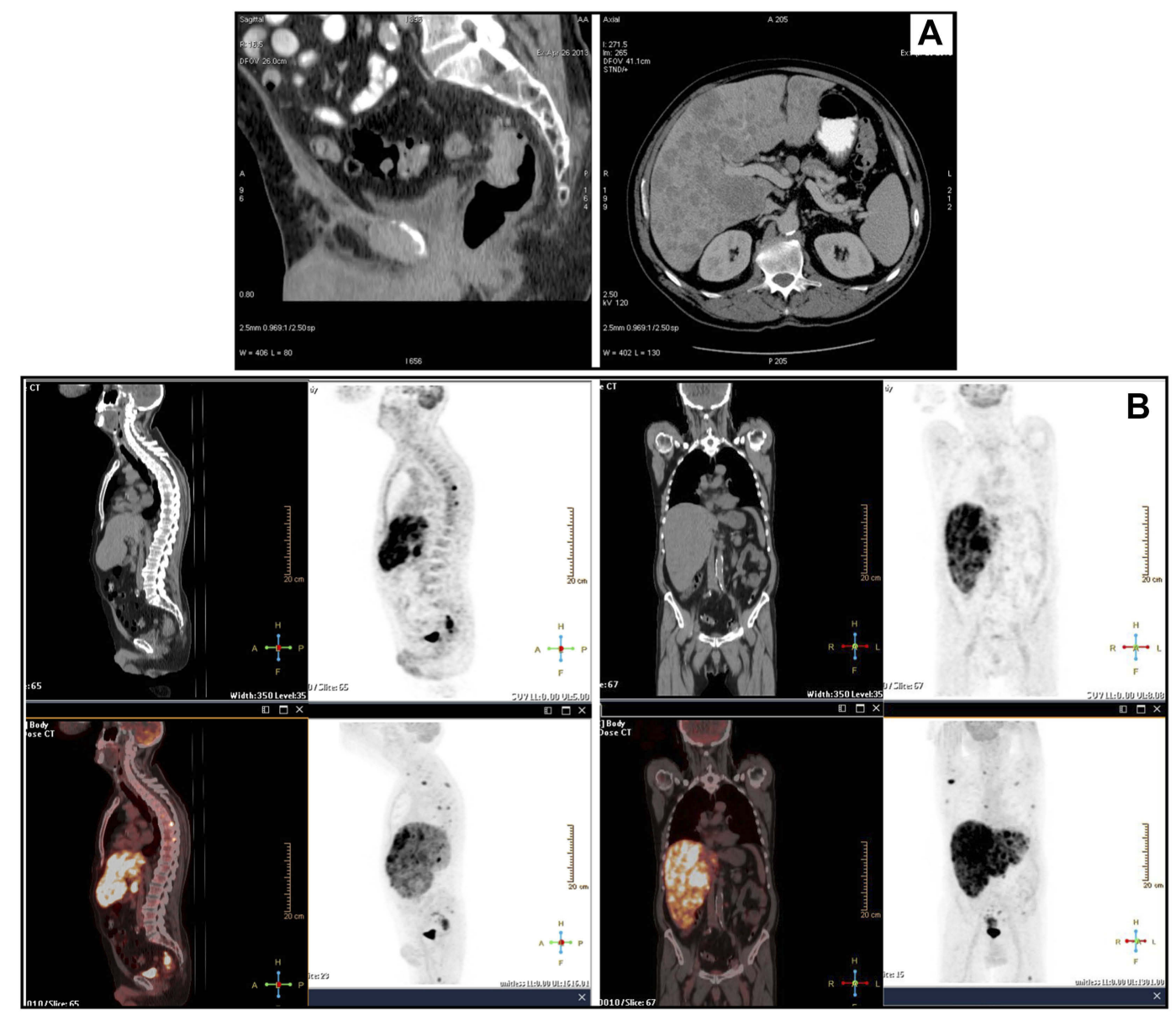

Figure 4 HGNEC CT scan (A) and CT PET (B) showed rectal tumor and liver metastases.

Abbreviations: HGNEC, high grade neuroendocrine rectal carcinoma; CT, computed tomography; PET, positron emission tomography.

poor prognosis, of colorectal cancer which were described for the first time by Clery et al in 1962 . They account for $<1 \%$ of all tumors of the large intestine. ${ }^{14,15}$ Because of the rarity, histological heterogeneity, and the poor prognosis, it is crucial to have a valid and rapid method for a proper diagnosis.

In this report we described a case of HGNEC analyzed for the first time, also with the pCLE system. The potential role of pCLE has been demonstrated in different pathologic conditions of the GI tract. ${ }^{9,10}$ Considering the prognostic implications, the possibility of diagnosing pre-malignant and malignant lesions through pCLE system can be very useful. Accurate diagnosis and staging are essential for therapeutic planning, particularly for aggressive tumors. All the studies performed with pCLE revealed the clinical usefulness and predictive power for in vivo diagnosis. ${ }^{12,16}$ In this case report we confirmed the usefulness of pCLE as a diagnostic method that also permits the discrimination of HGNECs from PDAs.

Although the innovative pCLE technology offers many advantages for clinical practice, the costs can limit its feasibility in the standard diagnostic procedure plan. Moreover, possible technical problems, such as bleeding during the endoscopic procedures, may impair image quality. This issue can be overcome by taking particular care to gently place the probe in contact with the tissue in order to avoid trauma. From an organizational set-up, the total endoscopic (endoscopy and pCLE) procedure obviously required a longer time than the standard method (only endoscopy) but also, this disadvantage could be of little significance considering the achievement of a real-time diagnosis. It should also be mentioned that the image interpretation obtained by pCLE recording requires an expert endoscopist fully trained in confocal endomicroscopy and tutorial meetings between endoscopists and blinded investigator discussing video sequences, images, and mosaicing pictures.

The finding that patient reported multiple liver metastasis is in accord with other studies describing colorectal HGNECs with metastatic disease, particularly in the liver, 
which is the main site of metastases for GI HGNEC. ${ }^{5,17}$ Cisplatin plus etoposide is the most utilized regimen. ${ }^{1}$

In conclusion and in our hands, pCLE represents a helpful tool for in vivo HGNEC diagnosis, allowing prompt and careful management of the patient. Obviously, to become solid and highly accurate, this technique should be performed on a group of HGNEC patients. In the absence of other cases enrolled in this study, since this type of tumor is extremely rare, especially in the rectum, ${ }^{4}$ at the moment we only suggest some pCLE criteria for the HGNEC evaluation. These deal with the presence of a huge amount of small cells and highly altered vasculature. Anyway, it should be retained that the innovative pCLE approach can improve the diagnostic accuracy and accomplish optimal clinical management of HGNEC patients.

\section{Ethics approval and informed consent}

The methodologies conformed to the standards set by the Declaration of Helsinki. The patients were enrolled within a study approved by the Institutional Board (ethical committee) of IRCCS- CRO, Centro di Riferimento Oncologico, Aviano, Italy (reference number CRO-201403). The patient provided written informed consent that included publication of the case details and accompanying images.

\section{Acknowledgments}

This work was supported by Minister of Health (reference number RF-2016-02361525) to Renato Cannizzaro and Paola Spessotto.

\section{Disclosure}

Professor Renato Cannizzaro reports grants from Ministry of Health Italy, during the conduct of the study. The authors report no other conflicts of interest in this work.

\section{References}

1. Smith J, Reidy-Lagunes D. The management of extrapulmonary poorly differentiated (high-grade) neuroendocrine carcinomas. Semin Oncol. 2013;40(1):100-108. doi:10.1053/j.seminoncol.2012.11.011
2. Oronsky B, Ma PC, Morgensztern D, Carter CA. Nothing but NET: a review of neuroendocrine tumors and carcinomas. Neoplasia. 2017;19(12):991-1002. doi:10.1016/j.neo.2017.09.002

3. Taal BG, Visser O. Epidemiology of neuroendocrine tumours. Neuroendocrinology. 2004;80(1):3-7. doi:10.1159/000080731

4. Bertani E, Ravizza D, Milione M, et al. Neuroendocrine neoplasms of rectum: a management update. Cancer Treat Rev. 2018;66:45-55. doi:10.1016/j.ctrv.2018.04.003

5. Smith JD, Reidy DL, Goodman KA, Shia J, Nash GM. A retrospective review of 126 high-grade neuroendocrine carcinomas of the colon and rectum. Ann Surg Oncol. 2014;21(9):2956-2962. doi:10.1245/s10434-014-3725-3

6. Sorbye H, Strosberg J, Baudin E, Klimstra DS, Yao JC. Gastroenteropancreatic high-grade neuroendocrine carcinoma. Cancer. 2014;120(18):2814-2823. doi:10.1002/cncr.28721

7. Kuiper T, van den Broek FJ, van Eeden S, Fockens P, Dekker E. Feasibility and accuracy of confocal endomicroscopy in comparison with narrow-band imaging and chromoendoscopy for the differentiation of colorectal lesions. Am J Gastroenterol. 2012;107(15720291):543-550. doi:10.1038/ajg.2012.14

8. Wallace MB, Fockens P. Probe-based confocal laser endomicroscopy. Gastroenterology. 2009;136(1509-0012):1509-1513. doi:10.1053/j. gastro.2008.11.049

9. Wang KK, Carr-Locke DL, Singh SK, et al. Use of probe-based confocal laser endomicroscopy (pCLE) in gastrointestinal applications. a consensus report based on clinical evidence. United European Gastroenterol J. 2015;3(2050-6406):230-254. doi: $10.1177 / 2050640614566066$

10. De Palma GD. Confocal laser endomicroscopy in the 'in vivo' histological diagnosis of the gastrointestinal tract. World $J$ Gastroenterol. 2009;15(1007-9327):5770-5775. doi:10.3748/ wjg. 15.919

11. Cannizzaro R, Mongiat M, Canzonieri V, et al. Endomicroscopy and cancer: a new approach to the visualization of neoangiogenesis. Gastroenterol Res Pract. 2012;2012(1687-630X):537170. doi:10.1155/ 2012/585674

12. Spessotto P, Fornasarig M, Pivetta E, et al. Probe-based confocal laser endomicroscopy for in vivo evaluation of the tumor vasculature in gastric and rectal carcinomas. Sci Rep. 2017;7(1):9819. doi:10.1038/s41598-01710963-1

13. Zhang YL, Bai L, Li Z, et al. Lower dose of fluorescein sodium is more suitable for confocal laser endomicroscopy: a feasibility study. Gastrointest Endosc. 2016;84:917-923.e5. doi:10.1016/j. gie.2016.05.011

14. Clery AP, Dockerty MB, Waugh JM. Small-cell carcinoma of the colon and rectum. A clinicopathologic study. Arch Surg. 1961;83:164-172. doi:10.1001/archsurg.1961.01300140006002

15. Shafqat H, Ali S, Salhab M, Olszewski AJ. Survival of patients with neuroendocrine carcinoma of the colon and rectum: a populationbased analysis. Dis Colon Rectum. 2015;58(3):294-303. doi:10.1097/ DCR.0000000000000298

16. De Palma GD, Staibano S, Siciliano S, et al. In vivo characterisation of superficial colorectal neoplastic lesions with high-resolution probe-based confocal laser endomicroscopy in combination with video-mosaicing: a feasibility study to enhance routine endoscopy. Dig Liver Dis. 2010;42(1878-3562):791-797. doi:10.1016/j. dld.2010.03.009

17. Brenner B, Tang LH, Shia J, Klimstra DS, Kelsen DP. Small cell carcinomas of the gastrointestinal tract: clinicopathological features and treatment approach. Semin Oncol. 2007;34(1):43-50. doi:10.1053/j.seminoncol.2006.10.022 


\section{Publish your work in this journal}

OncoTargets and Therapy is an international, peer-reviewed, open access journal focusing on the pathological basis of all cancers, potential targets for therapy and treatment protocols employed to improve the management of cancer patients. The journal also focuses on the impact of management programs and new therapeutic agents and protocols on patient perspectives such as quality of life, adherence and satisfaction. The manuscript management system is completely online and includes a very quick and fair peer-review system, which is all easy to use. Visit http://www.dovepress.com/ testimonials.php to read real quotes from published authors.

Submit your manuscript here: https://www.dovepress.com/oncotargets-and-therapy-journal 